\title{
Efficient Energy Load Distribution Model using Modified Particle Swarm Optimization Algorithm
}

\author{
Dr. T. Vijayakumar, \\ Professor, \\ Department of ECE, \\ GNIT, Hyderabad, India. \\ Email id: vishal_16278@yahoo.co.in
}

\author{
Mr. R. Vinothkanna, \\ Department of ECE, \\ Vivekanandha College of Technology for Women, \\ Namakal, India. \\ Email: rvinothkannaphd@gmail.com
}

\begin{abstract}
Reduction of emission and energy conservation plays a major role in the current power system for realizing sustainable socio-economic development. The application prospects and practical significance of economic load dispatch issue in the electric power market is remarkable. The various generating sets must be assigned with load capacity in a reasonable manner for reducing the cost of electric power generation. This problem may be overcome by the proposed modified particle swarm optimization (PSO) algorithm. The practical issue is converted and modelled into its corresponding mathematical counterpart by establishing certain constraints. Further, a novel interdependence strategy along with a modified PSO algorithm is implemented for balancing the local search capability and global optimization. Multiple swarms are introduced in the modified PSO algorithm. Certain standard test functions are executed for specific analysis. Finally, the proposed modified PSO algorithm can optimize the economic load dispatch problem while saving the energy resources to a larger extent. The algorithm evaluation can be performed using real-time examples for verifying the efficiency. When compared to existing schemes like artificial bee colony (ABC), genetic algorithms (GAs), and conventional PSO algorithms, the proposed scheme offers lowest electric power generation cost and overcomes the load dispatch issue according to the simulation results.
\end{abstract}

Keywords: Power generation, particle swarm optimization, load dispatch, genetic algorithm, artificial bee colony;

\section{Introduction}

The energy optimization issues have increased with the growing market demands and the resource conscious society. Low cost electricity generation and load dispatch issues need to be addressed to overcome these optimization issues [1]. Under various constraint conditions, least increase in the power system and generator cost can be ensured. Maintaining active power generation range, reducing power loss of the system and satisfying the electrical requirements of the users are some of the prominent constraints. The power system, its economic and safety benefits are affected by the energy load distribution issues. Several researchers and scholars are focused on reducing the cost of the power system and generator. Point-to-point scheme, sequential technique, equal incremental principle and Lagrange method are some of the traditional techniques for solving the energy load distribution issue. The diverse results and strong non-linearity are the major characteristics of the energy load distribution issue that cannot be addressed by the traditional schemes [2]. Utilization of intelligent optimization algorithms can solve these issues in a much efficient and reliable manner. This has grasped the attention of several researchers to work in this domain.

This paper presents a modified particle swarm optimization algorithm that can offer the lowest electric power generation cost and can overcome the load dispatch issue [3]. The mutual interdependence of biological populations and the influence they have on each other forms the basis of this algorithm. It consists of multiple swarms. The weight of the linear descending inertia of the PSO algorithm helps in optimization of the swarms through several iterations. The last swarm is used for self-updating the position information of the previous swarms. Rest of this paper is organized as follows. A detailed review of the existing schemes for overcoming the load dispatch issue is done in section two. Section three provides a detailed explanation of the proposed modified PSO algorithm with the required diagrams. Section four provides the results, analysis and discussion of the proposed scheme. Part five presents the conclusion and future scope of the work. 
Journal of Artificial Intelligence and Capsule Networks (2020)

Vol.02/ No.04

Pages: 226-231

http://irojournals.com/aicn/

DOI: https://doi.org/10.36548/jaicn.2020.4.005

\section{Literature Survey}

The load distribution issue is addressed using the differential artificial bee colony algorithm inspired by the differential evolution algorithm [4]. The search behaviour of the bees employed is improved by this algorithm while providing an efficient repair procedure. The load distribution issues in thermal power plants is overcome with the help of improved chaos particle swarm optimization algorithm. It works on the principle of chaos particle swarm optimization algorithm [5]. Chaotic sequence and parabola are used for generation of inertia weight while implementing the genetic algorithm with crossover operation for increasing the particles diversity in the swarm [6]. A combination of particle swarm optimization and differential evolution is used to create a novel hybrid algorithm for solving the load distribution issue [3]. Three swarms are involved in the population of this research. The efficiency and convergence provided this model is high. The hybrid algorithm addresses the economic dispatch issue with a new approach.

The harmonic search, artificial bee colony and ant colony optimization algorithms are combined to form a new framework to address the load dispatch issue [7]. This model offers minimal energy generation cost and optimal operational performance. The nonconvex economic load dispatch issues are addressed by a novel phasor particle swarm optimization algorithm. This system offers improved reliability and efficiency [8]. When compared to the existing algorithms, the lower computational cost and better solutions are provided by the Marine Predators Algorithm. Minimization of total active power loss and optimization of multi-objective function is performed with the help of an improved equilibrium optimization algorithm. The performance of distributed systems are enhanced with the help of a multi-objective swarm optimizer [9].

\section{Proposed Work}

The Particle Swarm Optimization (PSO) algorithm is similar to the other swarm intelligence based algorithms. It works on the principle of the predatory behaviour of birds. The PSO algorithm has improved structures and lesser parameters [10]. Further, the iterative rules of this algorithm are also relatively simple. The promotion and application of this algorithm is high in diverse fields. Position and velocity are the significant variables of PSO algorithm. The modified PSO algorithm offers simplicity of operation. Easy tapping of local optimum is an inevitable disadvantage of this algorithm unlike the other existing algorithms. The speed of convergence reduced in SOS algorithm at later stages [11]. This can be combined with the PSO algorithm to solve complex problems [12]. The nature of mutual interdependence forms the base strategy of the proposed modified PSO algorithm. During the complete iterative process, the particles support each other in this model that helps in achieving optimal individuals.

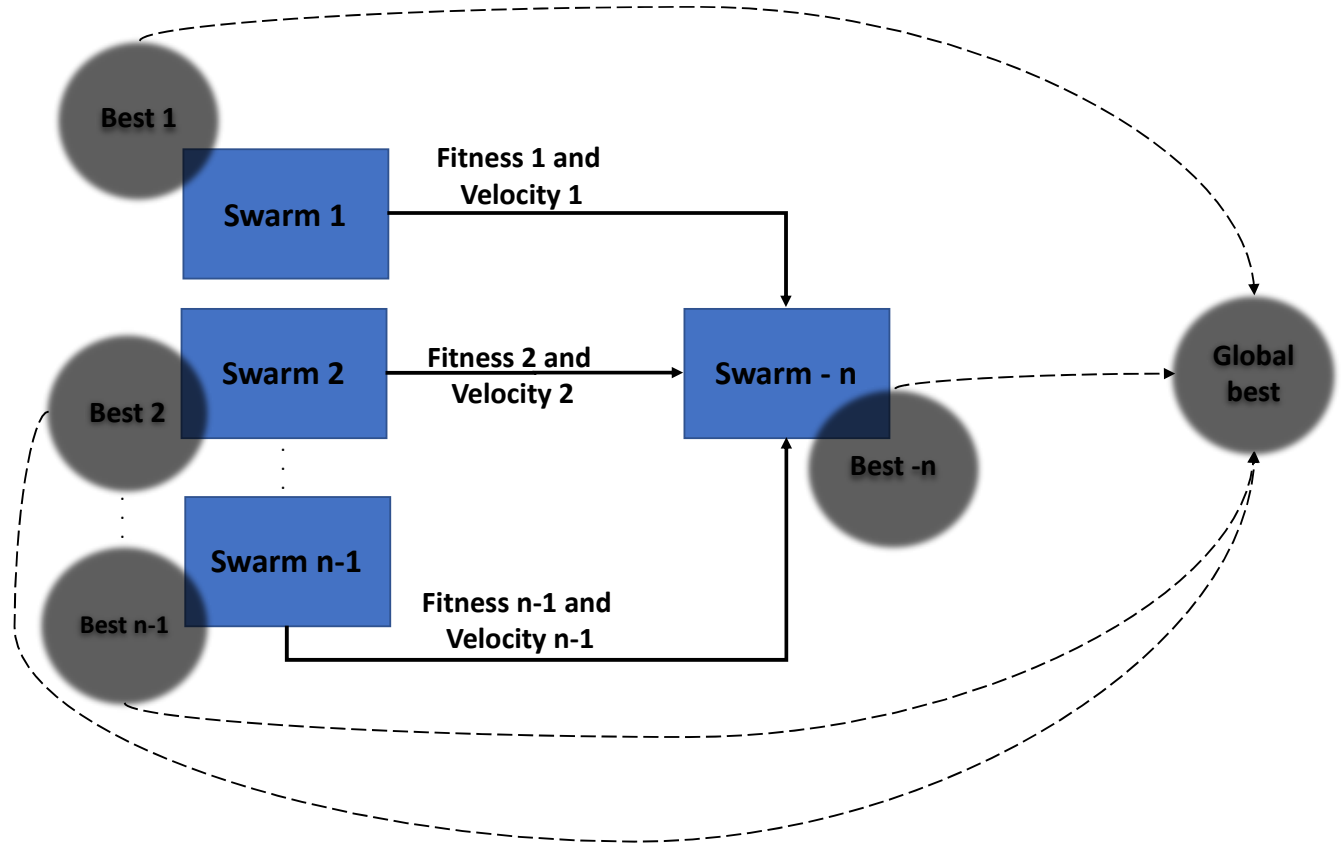

Figure 1: Strategy for selection of global best fitness value 
Journal of Artificial Intelligence and Capsule Networks (2020)

Vol.02/ No.04

Pages: 226-231

http://irojournals.com/aicn/

DOI: https://doi.org/10.36548/jaicn.2020.4.005

The evolutionary stagnation phenomenon can be prevented by the whole swarm using this technique. Figure 1 represents the concrete strategy of the proposed modified PSO algorithm. The iterative optimization is executed by the swarms independently. However, the fitness value is used for updating the velocity of the previous swarms in the last swarm. These multiple fitness values are finally considered to choose the global best value. The modified PSO algorithm can help solving the load distribution issue through the following procedure. Initialization of the position and velocity of all particles in the multiple swarms is performed using the following expression.

$$
\left\{\begin{array}{c}
x_{i, j}=\operatorname{rand}(N, 1) \times\left[\frac{\left(P_{\max }-P_{\min }\right)}{20}\right] \\
y_{i, j}=\operatorname{rand}(N, 1) \times\left(P_{\max }-P_{\min }\right)+P_{\min }
\end{array}\right.
$$

Where $\mathrm{j}$ ranges from 1 to $\mathrm{d}$ and $\mathrm{d}$ is the searching space dimension, $\mathrm{i}^{\text {th }}$ particle velocity is represented by $\mathrm{x}_{\mathrm{i}, \mathrm{j}} . \mathrm{i}^{\text {th }}$ particle position is represented by $y_{i, j}$. The $i^{\text {th }}$ generator's minimizing and maximizing active power is represented by $\mathrm{P}_{\min }$ and $\mathrm{P}_{\max }$ respectively. Random number between 0 and 1 is considered in $\operatorname{rand}(\mathrm{N}, 1)$. The functions position and velocity are used for calculation of the values of objective function of every particle with multiple iteration strategy. The proposed algorithm can be used for updating the functional parameters of all particles in the multiple swarms available. The procedure for updating these values can be represented by independent mathematical representations. Further, comparison of the output local optimal values obtained from each swarm is compared. The resultant global optimal value is obtained based on this comparison. Figure 2 shows the basic flowchart of the proposed modified PSO algorithm for solving the load distribution problem.

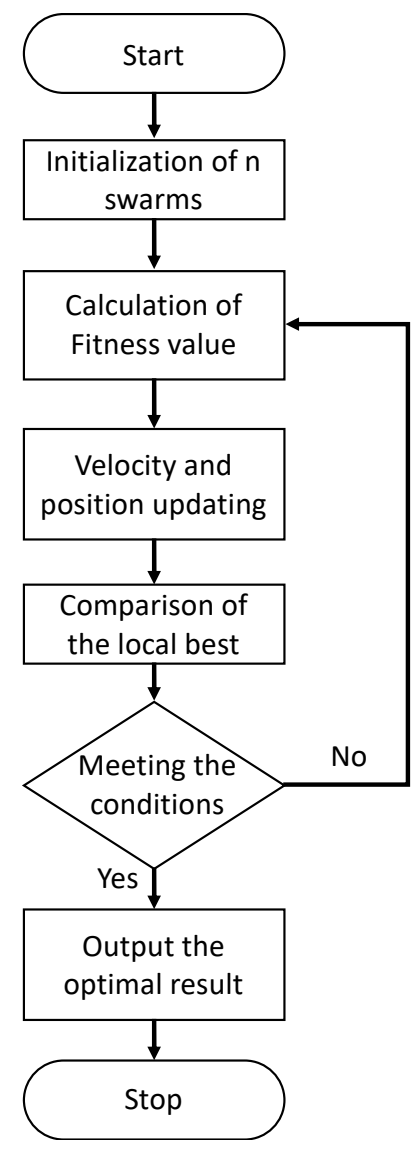

Figure 2: Basic Flowchart

\section{Results and Discussion}

Simulation and experimentation is performed with multiple practical examples for solving the load distribution issue. The proposed modified PSO algorithm is verified for its efficiency and validity. MATLAB environment is used for simulation of a 6-bus 3-machine system for experimental purpose and compared with the artificial bee colony (ABC), genetic algorithms (GAs), and conventional PSO algorithms along with the proposed 
Journal of Artificial Intelligence and Capsule Networks (2020)

Vol.02/ No.04

Pages: 226-231

http://irojournals.com/aicn/

DOI: https://doi.org/10.36548/jaicn.2020.4.005

modified PSO algorithm. Around 800MW estimated total load power is obtained from the entire system. Each generator is analysed and its consumption characteristic parameters as well as constraint conditions are estimated. The value-point effect of the first case is ignored while in rest of the cases, it is evaluated.

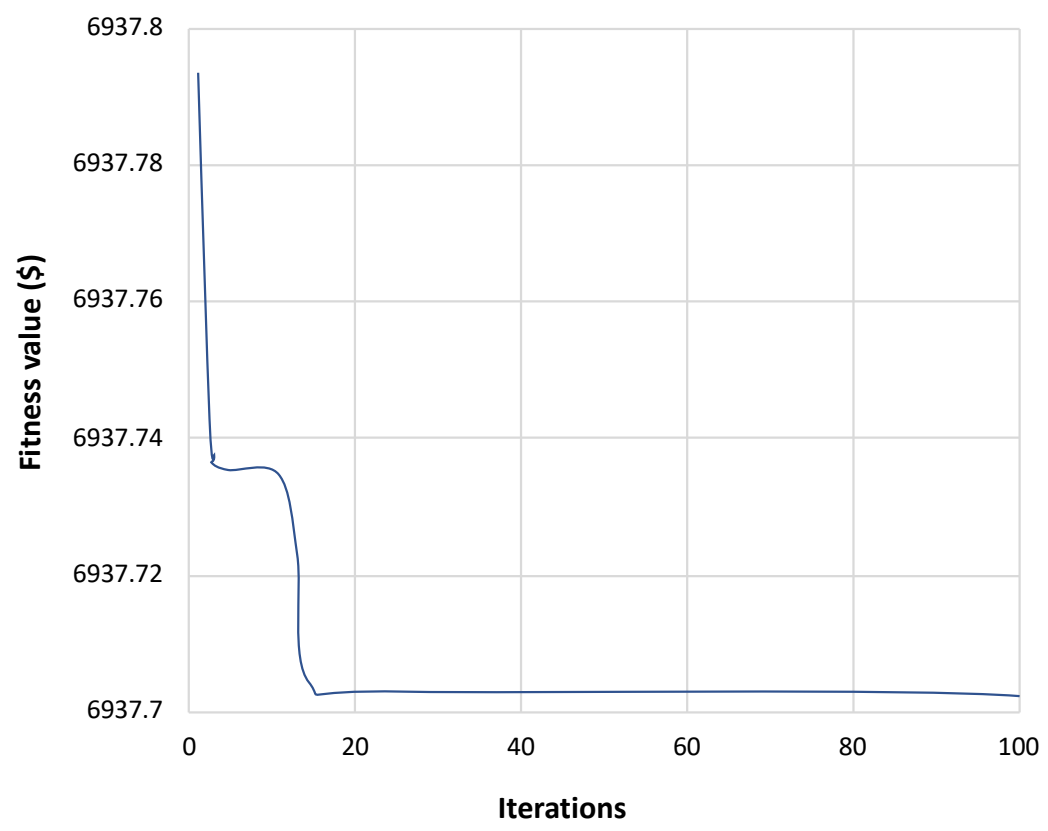

Figure 3: Evolutionary Process Curve of modified PSO algorithm

Proposed modified PSO algorithm is used for identification of optimal value through an evolutionary process as represented in figure 3. The curves of the evolutionary process through which optimal value is identified, is analysed for SOS, PSO and the modified PSO algorithm. Figure 4 represents the evolutionary process of the PSO algorithm. Further, ABC, GA, PSO and modified PSO algorithm are also compared. Based on analysis, it is evident that the optimal value is obtained at the earliest using the proposed modified PSO algorithm. High accuracy can be obtained as it can be isolated from the local optimum. The effect of valve-point is also eliminated by the proposed modified PSO algorithm thereby providing solution to the load distribution problem.

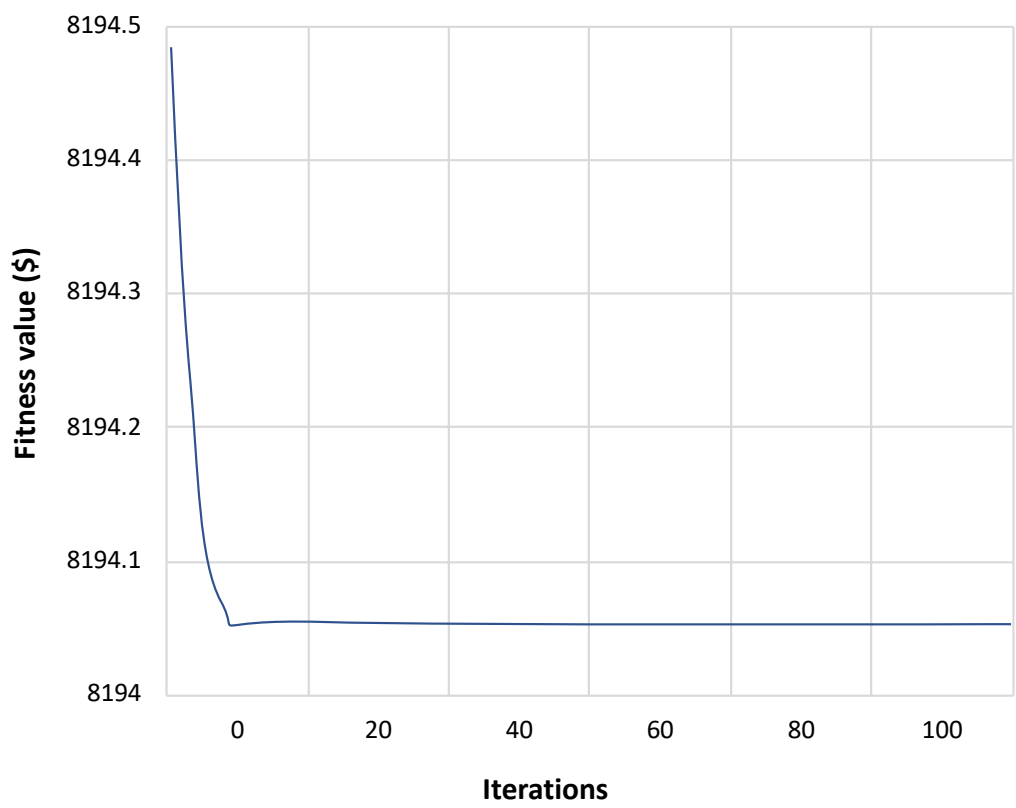

Figure 4: Evolutionary Process Curve of PSO algorithm

ISSN: 2582-2012 (online)

Submitted: 13.12 .2020

Accepted: 01.01.2021

Published: 08.02.2021 
Journal of Artificial Intelligence and Capsule Networks (2020)

Vol.02/ No.04

Pages: 226-231

http://irojournals.com/aicn/

DOI: https://doi.org/10.36548/jaicn.2020.4.005

Further, the curve of evolutionary process obtained by all four algorithms are compared and analysed as represented in figure 5. From this analysis, it is evident that the ability to identify the local optimum and efficient convergence rate obtained by the proposed modified PSO algorithm is high when compared to the ABC, GA and conventional PSO algorithms. 50 independent iterations are performed to obtain a fair conclusion and the objective due to the random initialization. Wire drawing is a significant valve-point effect that depends on the parameters representing the characteristics of energy consumption, which is overcome by the proposed algorithm.

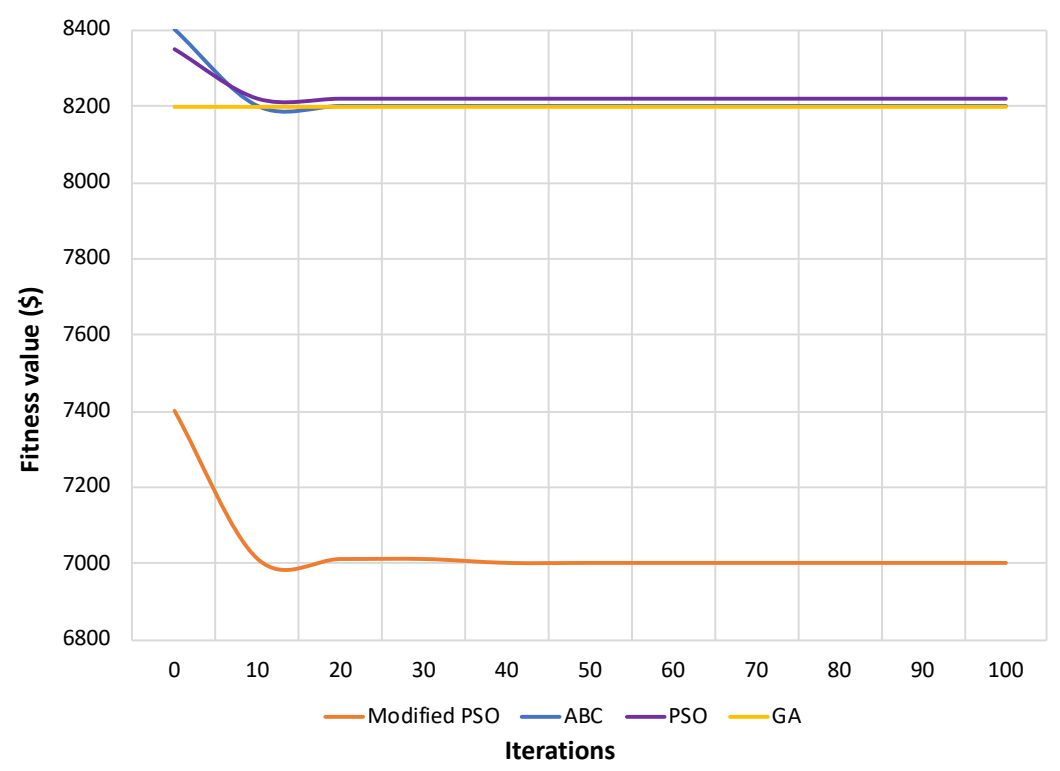

Figure 5: Comparison of modified PSO, ABC, PSO and GA algorithms

\section{Conclusion}

A modified PSO algorithm is proposed in this paper for providing lowest electric power generation cost and to overcome the load dispatch issue. The mutual interdependence of biological populations and the influence they have on each other forms the basis of this algorithm. It consists of multiple swarms. The weight of the linear descending inertia of the PSO algorithm helps in optimization of the swarms through several iterations. The last swarm is used for self-updating the position information of the previous swarms. Advanced convergence and other defects of the particles are improved by linearly decreasing the inertia weight. The practical issue is converted and modelled into its corresponding mathematical counterpart by establishing certain constraints. Practical examples and standard test functions are used for simulation, analysis and experimentation. The ABC, GA and conventional PSO algorithms are compared with the proposed modified PSO algorithm. The effectiveness of the proposed algorithm is illustrated in solving the load dispatch and power generation cost issues. Future work is directed towards further improvement of the algorithm to further optimize the efficiency of the model.

\section{References}

[1] Aghaei, J., Muttaqi, K. M., Azizivahed, A., \& Gitizadeh, M. (2014). Distribution expansion planning considering reliability and security of energy using modified PSO (Particle Swarm Optimization) algorithm. Energy, 65, 398-411.

[2] Abdelaziz, A. Y., Mohammed, F. M., Mekhamer, S. F., \& Badr, M. A. L. (2009). Distribution systems reconfiguration using a modified particle swarm optimization algorithm. Electric Power Systems Research, 79(11), 1521-1530.

[3] Eslami, M., Shareef, H., \& Mohamed, A. (2010, December). Optimal tuning of power system stabilizers using modified particle swarm optimization. In Proceedings of the 14th International Middle East Power Systems Conference (MEPCON’10), Cairo University, Egypt (pp. 386-391).

[4] Ferreira, J., Callou, G., \& Maciel, P. (2013). A power load distribution algorithm to optimize data center electrical flow. Energies, 6(7), 3422-3443.

[5] Cao, J., Li, K., \& Stojmenovic, I. (2013). Optimal power allocation and load distribution for multiple heterogeneous multicore server processors across clouds and data centers. IEEE Transactions on Computers, 63(1), 45-58. 
Journal of Artificial Intelligence and Capsule Networks (2020)

Vol.02/ No.04

Pages: 226-231

http://irojournals.com/aicn/

DOI: https://doi.org/10.36548/jaicn.2020.4.005

[6] Jiang, D., Zhang, P., Lv, Z., \& Song, H. (2016). Energy-efficient multi-constraint routing algorithm with load balancing for smart city applications. IEEE Internet of Things Journal, 3(6), 1437-1447.

[7] Paul, D., Zhong, W. D., \& Bose, S. K. (2016). Energy efficiency aware load distribution and electricity cost volatility control for cloud service providers. Journal of Network and Computer Applications, 59, 185-197.

[8] Chen, K., Lenhardt, J., \& Schiffmann, W. (2015, May). Improving energy efficiency of web servers by using a load distribution algorithm and shutting down idle nodes. In 2015 15th IEEE/ACM International Symposium on Cluster, Cloud and Grid Computing (pp. 745-748). IEEE.

[9] Arabali, A., Ghofrani, M., Etezadi-Amoli, M., Fadali, M. S., \& Baghzouz, Y. (2012). Genetic-algorithmbased optimization approach for energy management. IEEE Transactions on Power Delivery, 28(1), 162170.

[10] Rahim, S., Javaid, N., Ahmad, A., Khan, S. A., Khan, Z. A., Alrajeh, N., \& Qasim, U. (2016). Exploiting heuristic algorithms to efficiently utilize energy management controllers with renewable energy sources. Energy and Buildings, 129, 452-470.

[11] Smys, S. (2019). Energy-aware security routing protocol for WSN in big-data applications. Journal of ISMAC, 1(01), 38-55.

[12] Bhalaji, N. (2019). Delay diminished efficient task scheduling and allocation for heterogeneous cloud environment. Journal of trends in Computer Science and Smart technology (TCSST), 1(01), 51-62. 